19 Revue d'histoire du XIXe siècle

Société d'histoire de la révolution de 1848 et des

révolutions du XIXe siècle

$20 / 21 \mid 2000$

Varia

\title{
Anthropologie et progrès dans la philosophie de l'histoire d'Alphonse Esquiros. Le système des Fastes populaires
}

Loïc Rignol

\section{OpenEdition}

\section{Journals}

Édition électronique

URL : http://journals.openedition.org/rh19/214

DOI : $10.4000 /$ rh19.214

ISSN : 1777-5329

\section{Éditeur}

La Société de 1848

\section{Édition imprimée}

Date de publication : 1 juin 2000

Pagination : 157-179

ISSN : 1265-1354

\section{Référence électronique}

Loïc Rignol, «Anthropologie et progrès dans la philosophie de l'histoire d'Alphonse Esquiros. Le système des Fastes populaires », Revue d'histoire du XIXe siècle [En ligne], 20/21 | 2000, mis en ligne le 06 décembre 2016, consulté le 01 mai 2019. URL : http://journals.openedition.org/rh19/214 ; DOI : $10.4000 /$ rh19.214

Ce document a été généré automatiquement le 1 mai 2019

Tous droits réservés 


\title{
Anthropologie et progrès dans la philosophie de l'histoire d'Alphonse Esquiros. Le système des Fastes populaires
}

\author{
Loïc Rignol
}

\section{NOTE DE L'AUTEUR}

Ce travail émane des recherches initiées par l'équipe Littérature, idéologies, représentations, CNRS-Lyon 2 - Grenoble 3, dirigée par Philippe Régnier, sur la construction de la notion de race dans la littérature et les sciences humaines aux XVIII ${ }^{\mathrm{e}}$ et XIX ${ }^{e}$ siècles.

1 Pour Alphonse Esquiros (1812-1876), alors célèbre écrivain démocratique, auteur d'une récente Histoire des montagnards (1847), la crise profonde traversant la Monarchie de Juillet trouve son dénouement dans la révolution de 1848, porteuse des promesses de réconciliation et de fraternisation, avec laquelle doit s'ouvrir une ère d'unité. Il écrit pour prévenir tout retour des forces conservatrices : «Il s'agit, pour nous, de constituer l'unité humaine sur l'unité divine $»^{1}$. Aussi, avec l'abolition des titres et des privilèges, la révolution doit-elle intégrer également tous les citoyens sans distinction : «En France, il n'y aura aucun homme mis systématiquement en dehors de l'humanité $»^{2}$. Au point d'articulation des doctrines socialistes du temps, dites utopiques, et des réflexions contemporaines sur la science et la philosophie de l'histoire, Les fastes populaires, 1851-1853, offrent une vaste synthèse de la philosophie historique d'Esquiros, reprenant pour ce faire des développements antérieurs ${ }^{3}$. Elu à la Législative, représentant montagnard, il se propose de magnifier la marche triomphante du peuple dans l'histoire pour rendre compte de son émancipation progressive jusqu'à la Seconde République ${ }^{4}$. Le 
moment singulier de la révolution de 1848 se pense alors comme l'éclair de vérité, la révélation et le point d'accomplissement d'une logique sourde et profonde du Progrès universel. Se proclamant "maître d'école du peuple $»^{5}$, il pense que ce dernier ne pourra connaître et exercer pleinement ses droits nouvellement acquis que par la connaissance des luttes passées de ses ancêtres. À cet effet, il entreprend dans une fresque encyclopédique, mêlant la plupart des sciences naturelles de son temps, de jeter les bases d'une nouvelle philosophie de l'histoire, d'inspiration naturaliste et religieuse, Dieu gouvernant l'histoire par des lois naturelles. Sa théorie dessine trois cercles concentriques. Philosophe de la répétition de la nature dans l'histoire, il décrit d'abord un premier cercle, celui de la philosophie naturaliste de l'histoire fondée sur la science de l'univers en général et des races en particulier; dans un second cercle, il met en lumière, à la suite notamment des travaux de l'abbé Frère, la loi du progrès des races dans l'histoire, reproduction en abrégé de la perfectibilité universelle; enfin, explorant les fondements ethniques de la nationalité française, il peut, dans ce cadre général, expliquer l'histoire de la libération du peuple, descendant des Gaulois, par la dialectique sacrée du croisement des races.

\section{La philosophie naturaliste de l'histoire}

\section{L'idée d'une histoire universelle}

2 Alphonse Esquiros soutient que la connaissance objective des évolutions humaines doit procéder d'une réinscription de l'homme, du microcosme, dans l'univers, le macrocosme. Les principes de la philosophie de l'histoire doivent ainsi puiser leur rationalité et leur critérium de certitude dans les sciences naturelles. Il écrit: «Il résulte de ces idées générales que l'histoire proprement dite doit poser ses bases dans l'histoire naturelle $»^{6}$. La discipline historique doit ainsi s'unir à la physiologie pour découvrir un plan caché, une harmonie préétablie qui gouverne la succession des événements historiques et naturels. Il écrit: «Il faut qu'elle contracte avec les sciences naturelles et en particulier avec la physiologie une alliance étroite. De cette alliance sagement pratiquée, sortira la connaissance d'un ordre invariable de phénomènes sur lesquels les événements de l'histoire viendront, pour ainsi dire, se grouper. Là, mais là seulement, est le germe d'un perfectionnement nouveau dans les méthodes historiques ${ }^{7}$. Une seule loi régissant l'univers dans toutes ses parties, l'histoire et la nature se répondent, s'enchaînent : la seconde se répète dans la première, le cercle de l'histoire s'inscrit dans le cercle de la nature. Tout se correspond, se continue et se reproduit : «Partout nous vîmes se montrer avec éclat la répétition des mêmes phénomènes continués, et le maintien des mêmes lois. Les cercles s'élargissent ou diminuent; la vie oscille sous toutes les formes; mais le rapport des choses ne change pas ; c'est un beau motif de ravissement pour l'esprit que le spectacle de cette merveilleuse unité $! »^{8}$.

La science naturelle de l'histoire s'étend donc aux dimensions de l'univers, elle constitue une cosmologie : "Si la science dont nous essayons de poser les bases a un nom dans l'avenir, ce ne sera plus la philosophie, mais la cosmologie de l'histoire qu'il faudra dire ${ }^{9}$ . Un déterminisme implacable s'applique aux développements de l'espèce humaine. L'histoire des hommes, branche de l'histoire universelle, est soumise aux mêmes lois naturelles. Ce sont les décrets par lesquels la providence gouverne les transformations du monde : « Il y a dans les événements une force fatale et invincible qui les entraîne, malgré 
tous les efforts des hommes, au but que Dieu leur a marqué $»^{10}$. Aussi, découvrir l'ordre général qui préside à l'essor du globe et de l'humanité, c'est révéler la mission divine que le créateur leur a assignée : «Cette science constitue, si nous osons dire, la théologie de la nature $»^{11}$. La création fait en effet retentir la parole suprême du créateur, la nature s'honore/sonore de Dieu. La connaissance de l'histoire, appuyée sur les sciences naturelles, devient de ce fait sacrée, religieuse : «La croyance à une force insurmontable, qui pousse les événements vers un dessein supérieur, est la religion de l'histoire $»^{12}$. La philosophie de l'histoire d'Alphonse Esquiros, réconciliant la science, la nature et la religion sur la grande scène de l'univers, trouve son point culminant dans la pensée du progrès. Penser l'histoire, c'est mettre en lumière la loi objective et sainte qui préside aux transformations incessantes des êtres dans la création. Le savoir historique est science du progrès. Et c'est bien parce que les hommes se trouvent réinscrits, replacés dans la nature, que l'on peut découvrir la règle nécessaire qui préside au perfectionnement des civilisations. Esquiros le soutient, c'est bien la biologie de l'humanité qui la rend essentiellement progressive. Sans nature, pas de perfectibilité : «Comment introduire la certitude dans la philosophie de l'histoire ? En découvrant le principe moteur qui dirige le progrès des nations et de l'humanité. Ce principe est dans la nature $»^{13}$.

4 Le progrès est la loi même du monde, il est universel : «Or, qu'est-ce que le progrès ? Il faut entendre par ce mot la marche générale du monde vers un état de choses perfectionné, auquel tendent d'un effort unanime la création et l'humanité $»^{14}$. Aussi, les conservateurs ne peuvent-ils arrêter le cours de l'histoire. Leur combat est inutile. Puisque la nature est elle-même progressive et révolutionnaire, il faudrait détruire l'ordre du monde et la nature même de l'être humain pour en arrêter le mouvement. En d'autres termes, il faudrait défier la volonté de Dieu dont ils émanent. Toute résistance aux progrès de la création constitue aussitôt un blasphème contre le créateur: «La nature est révolutionnaire. Il faut en prendre votre parti ; tout ce que vous ferez contre le mouvement, vous le ferez contre l'ordre établi de Dieu [...]. Quand vous attaquez l'esprit révolutionnaire, ne vous en prenez donc plus aux hommes de 89 ni de 93 ; prenez-vous en à la nature ; accusez les lois générales de la création ; accusez Dieu $! »^{15}$.

5 Cette énergie objective et divine animant l'univers et l'histoire trouve son expression dans l'anthropologie des races. Celles-ci constituent les forces orientant la destinée des peuples. La science de l'histoire est science des races, répétant en abrégé dans les lois de leur organisation, les puissances cosmiques qui commandent le monde et ses transformations.

\section{La résurrection et la monadologie des races}

6 Le principe proclamé d'un retour à la nature se traduit, pour l'être humain, par une volonté de penser sa propre nature, de se saisir comme un être appartenant à une communauté jugée naturelle : la race. Au début du XIX ${ }^{e}$ siècle, la notion se répand bien audelà des cercles des naturalistes. Elle devient rapidement à la mode. Penser la race, c'est d'abord proposer un retour à soi pour l'humanité, c'est-à-dire replacer les peuples dans les cadres que la nature leur a tracés. Chaque nation doit, par conséquent, revenir à sa propre essence. Elle doit prendre conscience d'elle-même et se reconnaître comme appartenant à une race, partie intégrante de la famille humaine. Cette thématique se décline en deux moments: le retour comme réveil, sortie de l'oubli, le retour comme 
réconciliation avec soi-même, c'est-à-dire comme réappropriation démocratique de son identité.

Le retour à la race se présente d'abord comme une résurrection, un retour à la vie. En effet, on considère souvent au $\mathrm{xIX}^{\mathrm{e}}$ siècle que les ethnies constituent une réalité inaltérable et éternelle. Si les hommes meurent, les entités naturelles auxquelles ils appartiennent, espèces ou races, sont, elles, toujours jeunes. Les nations, impérissables dans l'histoire, constituent un authentique monument qu'il importe de tirer de l'oubli. Cet édifice naturel, d'autant plus difficile à étudier qu'il est proche, nécessite pour être étudié une conversion des regards : l'humanité doit se constituer en sujet et objet de savoir. C'est pourquoi, ainsi que le rappellent les anthropologues de ce temps, l'homme est l'être qui fut observé le dernier: observant les autres créatures, il s'était effacé et oublié devant l'objet de ses contemplations. "L'anthropologie est une science cultivée depuis peu [...] $\aleph^{16}$, déclare William-Frédéric Edwards (1777-1842), fondateur en 1839 de la Société ethnologique de Paris. Alphonse Esquiros le précise à son tour: "Il nous faut déplacer le terrain des observations historiques. Jusqu'ici on n'a cherché le progrès que dans les développements du droit, de la science, des arts, des doctrines, des croyances religieuses. Il est un autre ordre de monuments qu'on a négligé de consulter. Le premier de ces monuments, c'est l'homme $\aleph^{17}$. Si les races et les espèces sont immortelles, le XIX ${ }^{\mathrm{e}}$ siècle apparaît comme le moment de leur réapparition, de leur réveil, annonciateur de leur retour sur la scène de l'histoire ${ }^{18}$.

Dès lors, le réveil des nations s'ouvre vers la réconciliation. L'invocation des races, au siècle dernier, a longtemps sous-tendu des revendications démocratiques : la sortie du sommeil des peuples exigeant leur liberté et l'exercice de leur souveraineté. Proclamer son appartenance à une race, c'était se penser membre d'une association naturelle et historique, la partie intégrante d'un sujet collectif revendiquant son émancipation. Le printemps des peuples de 1848 illustre avec éclat ce réveil des nations et des races en Europe. L'appel à la race devient alors synonyme d'appel à la démocratie ${ }^{19}$. Cette affirmation de soi d'une nation, se pensant elle-même dans ses caractères anthropologiques, fonde ainsi une union, une association entre les membres de cette communauté. Cette réconciliation avec autrui jette les bases d'une politique. Mais audelà, dans une dimension plus éthique et religieuse, elle suppose que chacun s'assume comme être naturel, comme individu dans cette communauté. En surmontant l'anathème jeté contre la chair, la répudiation de la nature prononcée par le christianisme, il s'agit de surmonter l'opposition entre l'esprit et le corps, instance du péché, pour se ressaisir comme être indistinctement physique et moral. On comprend pourquoi les saintsimoniens, adeptes d'une réhabilitation de la chair, se soient intéressés de près à l'ethnologie, entendue comme science des races. La réconciliation politique avec autrui se double ainsi d'une réconciliation éthique avec soi- même.

9 Au point de convergence de ces considérations anthropologiques, politiques et morales, émerge donc la notion de race. Le saint-simonien Euryale Cazeaux (1805-1880), pour en marquer le rôle pivot, la qualifie de "monade ", c'est-à-dire, dans la philosophie de Leibniz, la substance simple, première, indivisible et fondamentale dont les êtres sont composés : « la monade race, dis-je, ne pourrait se soustraire longtemps aux efforts réunis des études historiques et de l'appréciation des faits $»^{20}$. Elle devient la figure centrale de l'histoire. En premier lieu, l'écriture de l'histoire prend, avec cette nouvelle notion, une autre orientation politique. Elle ne se pose plus comme l'histoire dynastique des « races royales" ou des lignages aristocratiques. Elle devient le récit démocratique de 
l'émergence du peuple, des masses oubliées et méprisées par l'historiographie. Les races doivent naitre dans le discours de l'histoire parce qu'elles ont ressuscité dans le monde de l'histoire. Esquiros le souligne: "Jusqu'à présent on a écrit l'histoire des rois, des hommes célèbres, des faits éclatants, des événements qui ont élevé la grandeur ou précipité la ruine des nations, des conquêtes et des fastes militaires; on a oublié l'histoire de la multitude $»^{21}$. En d'autres termes, il s'agit de constituer la «république de l'histoire ».

En second lieu, la monade race est pensée comme une force nécessaire qui resurgit perpétuellement. La race enferme en elle la loi nécessaire de la destinée des peuples. Les ethnies portent en leur sein des instincts, c'est-à-dire des impulsions aveugles, inconscientes et nécessaires qui les poussent à l'action. Ces forces définissent leur degré d'aptitude aux arts, aux sciences, au commerce et à l'industrie. L'ethnologie établit ainsi l'horoscope de leurs destinées en diagnostiquant leurs capacités natives se déployant inéluctablement au fil des siècles ${ }^{22}$. De ce fait, la science de l'histoire doit s'orienter vers une sorte de monadologie historique, description de ces puissances que sont les races, définissant le sens et la trajectoire qu'elles empruntent dans le temps: «Dans la philosophie de l'histoire, il serait nécessaire qu'un grand et sage esprit vînt substituer la notion de force à la notion de substance, et fit une application des doctrines leibnitziennes aux faits historiques. Au lieu de cette unité confuse et de cette fatalité logique dont nous entretiennent les modernes théoriciens, qui donc nous établira la hiérarchie préétablie et nous déroulera le combat providentiel de ces forces premières appelées caractères, instincts des races, et d'où découle l'histoire? Qui nous donnera une monadologie historique? $\aleph^{23}$.

11 Enfin, si la philosophie historique des races introduit dans ses études le concept de force, inéluctable et nécessaire, celle-ci revêt le caractère du progrès. Les énergies matérialisées dans les races répètent, en réduction, les lois générales orientant la création depuis ses origines vers le perfectionnement. L'anthropologie des races constitue le cadre de l'amélioration des sociétés et des civilisations. La nature des hommes porte en elle le mouvement même de perfectibilité de l'humanité, que rien ne peut entraver. La physiologie des races constitue en ce sens le moteur de la progression humaine. Alphonse Esquiros écrit: "Il existe pour les nations une croissance naturelle. La civilisation, envisagée au point de vue scientifique, est le résultat de forces qui se développent chez les hommes réunis en société. Ces forces sont déterminées par un principe moteur et par une loi. Ce principe moteur réside dans l'organisme des races, qui, sous l'influence du temps et du milieu social, se modifie séculairement et périodiquement. La loi est fixée par les combinaisons de chaque âge social avec les éléments extérieurs du progrès $»^{24}$.

12 Cette loi organique du progrès, Esquiros la découvre dans les recherches de l'abbé Frère (1786-1858), dont il fut l'élève, et qu'il commenta à de nombreuses reprises. Elles lient, d'une manière étonnante, la science de l'homme, l'histoire des sociétés et les révélations religieuses dans une forme d'anthropologie sacrée de l'histoire. 


\section{L'abbé Frère et le déchiffrement anthropologique du progrès des races}

\section{Les hiéroglyphes naturels de la perfectibilité humaine}

${ }^{25}$ Alphonse Esquiros a longuement commenté et médité les Principes de la philosophie de l'Histoire de l'abbé Frère, publiés en 1838. Il y voyait « le germe d'une révolution pour la philosophie de l'histoire ${ }^{26} \mathrm{Il}$ regrette en effet que les races n'aient été étudiées, par les naturalistes, que d'un point de vue stationnaire, c'est-à-dire en comparant les races entre elles, à partir de leurs boîtes crâniennes notamment, sans rapprocher les évolutions d'une même race au cours des siècles: "La physiologie comparée d'une même race aux divers âges de son développement social, n'a pas été faite jusqu'ici : c'est à cette étude que nous vous convions $\aleph^{27}$. L'anthropologie comparée d'une race aux divers âges de son développement constitue précisément la grande invention de l'abbé Frère. De même que chaque individu déploie ses forces physiques, intellectuelles et morales d'âge en âge, chaque nation emprunte une voie nécessaire pour accomplir ces mêmes progrès : la vie de l'organisation sociale correspond à celle de l'organisation individuelle.

Ainsi, chaque nation, après sa sortie du nomadisme, obéit-elle à un progrès dans ses aptitudes suivant une série de sept périodes, à l'image de la croissance de chaque individu, obéissant, d'après l'abbé Frère, à sept séquences de sept $a n s^{28}$. Chaque âge de la civilisation se compose de 233 ans. L'être humain se trouve donc soumis à la contrainte d'une loi régissant son évolution organique, sociale et psychologique. L'homme, dans chacune de ces périodes, se caractérise par des capacités spécifiques, une organisation physique et des maladies dominantes, une forme particulière d'organisation sociale, d'institutions et de mœurs. Par conséquent, dans le système philosophique de l'abbé Frère, l'homme se trouve déterminé, à son insu, par la loi naturelle du progrès, voulue par Dieu : « L'homme, suivant ce système, n'est pas absolument maître de la direction de son esprit; il vit sous la dépendance des organes, des dispositions morales, des capacités propres à son âge viril et à son âge social. Dieu lui-même enferme son intervention dans ces lois du progrès qui régissent la nature humaine $»^{29}$. C'est la nature qui veut le progrès des races et c'est ce dernier qui détermine le progrès des sociétés.

Après avoir posé le principe d'une puissance interne développant les facultés d'un peuple au cours des siècles, que l'on peut mathématiquement calculer, l'abbé Frère soutient la possibilité d'interpréter, à surface du crâne, les signes visibles de cette évolution physique et morale $\mathrm{e}^{30}$. Alphonse Esquiros commente l'intuition de son ancien professeur : «On entrevoit d'ici l'idée de $\mathrm{M}$. Frère : le progrès social n'est à ses yeux qu'une suite de périodes dont chacune marque son empreinte sur la tête de l'homme $»^{31}$. Disciple de Gall (1758-1828) et de Spurzheim (1776-1832), dont il partageait les principales convictions phrénologiques, «il croyait avec ses maitres que les diverses manifestations de l'intelligence, des sentiments ou des instincts sont liées à une certaine configuration du cerveau, principal organe de l'âme $»^{32}$. En effet, l'organisation cérébrale pour Gall est le grand livre dans lequel le savant peut lire les aptitudes des hommes et des animaux gravés dans les circonvolutions du cerveau, elles-mêmes façonnant l'enveloppe osseuse : "C'était pourtant, à son avis, le livre où Dieu avait scellé le mystère de la vie humaine " 33 . La tête devient parchemin, le crâne et ses configurations dessinent les lettres et les signes des facultés. Ils deviennent un document historique capital. Une réalité matérielle et 
anodine, une chose de peu : la structure osseuse, se trouve par-là érigée à la dignité de monument. La boîte crânienne devient l'inscription ésotérique des destinées humaines : «Le crâne, qu'on envisageait jusqu'ici comme une dépouille stérile, est, aux yeux de cette nouvelle science, un monument du progrès des races $»^{34}$. La structure de l'encéphale constitue un cartouche de hiéroglyphes à décrypter: la science historique devient sémiologie anthropologique. La science de l'homme fonde désormais la science de son histoire. Ainsi, l'abbé Frère a-t-il réuni, dans une collection, des crânes de chaque période, « il nomme cette série de types son alphabet : chacun de ces crânes, qui caractérisent une des formes et une des époques de la civilisation, est en effet un hiéroglyphe à l'aide duquel l'homme qui sait lire cette écriture peut reconstituer l'ensemble des aptitudes propres aux différents âges des sociétés $»^{35}$. Le musée de Gall représente, comme la collection craniologique de l'abbé Frère, un résumé de l'histoire contemporaine, écrit en traits d'os dans les dépouilles des hommes : «Le crâne était aux yeux de Gall une page solide sur laquelle la nature avait tracé en traits reconnaissables le caractère et le génie de chaque homme. Il prétendait donc qu'on pouvait reconstruire l'histoire d'un individu éteint, d'après les seules indications de la tête $"^{36}$. À l'aide de ces fragments, un naturaliste pourrait recomposer la vie entière des personnes et des sociétés dans lesquelles elles ont vécu. Le crâne est un langage parlant des hommes à leur insu. Il raconte leur histoire. Il s'offre comme la synecdoque des civilisations, la médaille oubliée qui les raconte pour ceux qui savent en déchiffrer l'écriture à demi effacée : «Se promener dans le cabinet du docteur Gall, c'est passer en revue une partie de l'histoire de ces dernières années, écrites en caractères indélébiles sur le crâne des hommes qui en ont composé les principaux éléments [...]. Quelque Cuvier à venir pourra, à l'aide de ces fossiles historiques, reconstruire l'image vivante de notre société, avec ses monstres et ses prodiges, ses révolutions et ses cataclysmes ${ }^{37}$.

Le progrès devient visible et palpable sur l'enveloppe crânienne, partant de la nuque pour aller vers le front : il manifeste une rationalité et une direction observables. Le sens de l'histoire d'une race se trouve gravé dans l'os de sa tête ${ }^{38}$. La série des crânes d'une même société résume, matériellement, son évolution. Esquiros écrit : «Cette échelle sériaire des développements organiques apportés au crâne par le mouvement de la civilisation, en dit plus que les plus éloquentes paroles : le progrès est désormais un fait palpable, moulé par Dieu sur la tête de l'homme ${ }^{39}$. Le progrès est devenu une force objective, animant les corps, et les poussant, à l'insu des individus, dans la voie de la perfectibilité biologique, sociale et morale. Esquiros le proclame avec lyrisme: «Le vieux monde théologique trembla d'épouvante sur ses bases ruinées, le jour où Galilée s'écria : La terre se meut, la terra si muove!' Que deviennent aujourd'hui les doctrines d'immobilité sociale et religieuse, devant la découverte de cette loi : la nature humaine se meut; l'organisation des peuples est soumise comme celle des individus à une croissance qui prend ses règles dans le temps. Les germes de la perfectibilité humaine et sociale des nations ont été déposés dans les caractères des races: ces germes se développent spontanément et nécessairement. - Que nous chantent après cela les partisans du statu quo, quand ils viennent nous parler de compression! Le mouvement n'est pas seulement hors de nous; il n'est pas même dans la partie libre de notre être, dans notre intelligence et dans notre volonté; non, il est encore dans nos organes; il est écrit dans notre chair, dans notre sang, dans notre système nerveux, dans les profondeurs de notre cerveau. Vous auriez détruit le progrès dans le monde à coup de canon; vous l'auriez enchaîné, musclé, garrotté dans des liens de fer, ce dieu mobile est insaisissable, que vous le retrouveriez 
encore dans votre propre nature. Cette force détestée, incompressible, fatale, elle n'est pas dans les doctrines socialistes : elle est dans l'homme ${ }^{40}$.

Tous les conservatismes sont donc condamnés par avance, dit Esquiros, rien ne pourra arrêter cette dynamique du perfectionnement des races. Pour la freiner, peut-être faudrait-il, à l'instar des Caraïbes, enserrer le cerveau des enfants dans des planches de fer ; mais ces tentatives resteraient vaines, « vos instruments de torture seraient vaincus par la force occulte de la nature, vis insista naturae; vos bourrelets de fer éclateraient sous les développements irrésistibles de la tête humaine $»^{41}$.

\section{« Bio-graphie » et blason des races dans l'histoire}

Avec les travaux de l'abbé Frère, on peut lire à la surface du crâne, de période en période, le progrès naturel des races, conditionnant leur progrès social. Avec cette alliance entre la physiologie et l'histoire, l'histoire s'inscrit dans l'histoire naturelle. La science de l'histoire est devenue, au sens strict du terme, une science d'observation et de déchiffrement : «Cette science tout nouvelle pourrait être définie : l'art de déchiffrer sur l'enveloppe osseuse du cerveau la chronologie du progrès $»^{42}$. On peut observer pour lire l'écriture hiéroglyphique des corps et prouver, par ce biais, ses assertions. On peut toucher du doigt, au sens strict du terme, les signes ossifiés du progrès. La discipline historique n'a plus seulement affaire à des documents écrits laissés par les hommes, vestiges du temps, mais peut recourir à un autre ordre de preuves matérielles, racontant la vie des morts. Alphonse Esquiros a souvent mis en opposition ces deux types d'archives. Il oppose en effet, dans une sorte de balancement, les sources, au sens large, fournies par une époque, restes des civilisations passées: chartes, édits, parchemins, monuments, œuvres d'art, médailles, etc., aux sources de la nature elle- même, c'est-àdire «l'étude des caractères physiques, ce langage de la nature ${ }^{43}$. Dans cette mise en parallèle, il accorde toujours la préférence aux signes naturels et anthropologiques des peuples contre les témoignages laissés par leur société. La science muette des livres doit en effet céder le pas devant les faits eux-mêmes parlants, ces traits de la nature constituant déjà, et avant toute verbalisation humaine, un langage : «Mes impressions furent plus vives encore, quand, passant de cette science muette des livres aux faits euxmêmes, aux faits parlants, [...] $»^{44}$. Les deux formes d'archives, les signes anthropologiques des corps et les œuvres écrites léguées par l'histoire révèlent donc l'existence de deux langages à partir desquels l'historien peut décrire l'évolution des civilisations. Avec l'abbé Frère et les travaux historiques des anthropologues, tels Edwards et Courtet de l'Isle par exemple, l'histoire des hommes se découvre dans leurs manifestations physiologiques: les corps sont devenus corpus. Ce langage de la nature, plus authentique et seul infaillible, doit servir de fondement à l'écriture de l'histoire. On peut bien parler, avec cette nouvelle forme d'histoire, d'une transformation épistémologique du discours historique, se découvrant une nouvelle positivité: les caractères observables des races, une nouvelle rationalité et un nouveau sens, le déchiffrement des signes naturels des corps. Mais cette mutation épistémologique est inséparable d'une inflexion politique du discours historique. Ce nouveau langage de l'histoire se double d'une nouvelle politique. L'analyse historique ne déchiffre plus le même blason, elle change de fondement et s'ouvre à la multitude.

Lorsque les physiologistes prétendent décrire l'histoire des nations et des races par l'inspection des formes crâniennes, ils érigent ces pièces anatomiques au rang de blason. 
Les aptitudes diverses des hommes se trouvent inscrites dans les bosses, dénivelés et protubérances des os du crâne. Ce langage objectif, c'est-à-dire de l'objet lui-même, devient le nouvel emblème, le nouveau drapeau des âges démocratiques, détrônant les blasons de la noblesse. Au Moyen Âge et sous l'Ancien Régime, le blason des familles royales et aristocratiques symbolise en effet leurs vertus, leurs aptitudes et leurs qualités éminentes, leurs titres de noblesse. Esquiros montre l'importance sémiologique des écussons et des emblèmes de cette époque. Par leur intermédiaire, les élites manifestaient leur supériorité et leur ancienneté aux yeux du peuple: «La naissance du blason se trouve étroitement liée à la noblesse de sang, qu'elle a, pour ainsi dire, mission de faire paraitre au- dehors, jusque sur le costume, par des signes de convention $»^{45}$. Le blason raconte l'histoire d'une dynastie ou d'un lignage, leurs faits d'armes, leurs raisons d'être et leurs titres de gloire. Il s'offre comme une généalogie allégorique. Les hommes et les femmes qui les portent s'inscrivent dans une histoire immémoriale, symboliquement contée par ces emblèmes leur imposant d'imiter les vertus de leurs aïeux. Dépositaires de traditions séculaires les précédant et devant leur survivre, les nobles pensent échapper à l'emprise du temps.

20 De ce fait, le blason devient un code, un langage ${ }^{46}$. Esquiros le soutient : « Le blason est la langue de la féodalité au Moyen Âge $~{ }^{47}$. Au XIX ${ }^{e}$ siècle, ces symboles d'une élite sont remplacés par un blason, nouveau et étonnant, plus populaire surtout : le crâne. Celui-ci est pensé comme l'étendard des âges démocratiques. Les armoiries des races nobles et royales sont supplantées par les armoiries, écrites par la main même de la nature, que désormais, chaque individu, puissant ou misérable, noble ou roturier, riche ou pauvre, arbore, inconsciemment, dans la forme naturelle de son encéphale. Aussi, la phrénologie, en décodant cette langue naturelle et symbolique de la tête, décerne-t-elle les véritables quartiers de noblesse en distinguant les authentiques capacités. Tout individu porte en lui, dans la forme de son crâne, les insignes de son talent, les emblèmes de ses mérites. Les écussons sont devenus crâniens. La phrénologie devient la science héraldique du cerveau à l'ère des masses. Alphonse Esquiros montre que les aptitudes inégales des individus, fondements de leur aristocratie naturelle, se lit désormais dans les hiéroglyphes du cerveau : «L'ensemble des facultés est circonscrit d'avance dans chaque individu, au point juste où Dieu a voulu l'arrêter. Gall ajoutait, au grand scandale du monde savant, que cette destination particulière à chacun était inscrite en caractères visibles sur la boîte osseuse du cerveau. Le crâne était, aux yeux du docteur allemand, un blason sur lequel la nature a marqué de sa main puissante les quartiers de noblesse de tous ses enfants. Cette noblesse-là est indélébile, car elle vient de Dieu et elle va à la société qui ne pourrait jamais subsister sans elle. C'est la variété nécessaire à l'unité de la race humaine $»^{48}$.

21 La langue naturelle du blason des crânes témoigne donc, dans son déplacement sémiologique, d'une évolution démocratique. Il ne s'agit plus de retracer, dans le langage des symboles féodaux, l'histoire des races nobles et royales, mais de découvrir, dans les emblèmes crâniens des races, le langage objectif et ignoré racontant leurs progrès. Les blasons artificiels des élites doivent disparaître devant les blasons naturels répandus dans la multitude. Le concept de biographie semble résumer cette double évolution épistémologique et politique. Longtemps en effet l'histoire d'un pays s'est résumée au récit des actes héroïques et des règnes des grandes figures du royaume. On relatait la biographie des rois et des nobles, on faisait la biographie du pouvoir dit Saint-Simon (1760-1825) : «Jusque vers le milieu du dernier siècle, l'histoire n’a presque jamais été 
qu'une Biographie du pouvoir, dans laquelle les nations ne figurent que comme instruments et comme victimes, et où se trouvent clairsemées çà et là quelques notions épisodiques sur la civilisation des peuples $»^{49}$. Après la Révolution française, il s'agit de substituer à la biographie des grands la biographie du peuple, selon la propre expression d'Esquiros. Ce déplacement démocratique du personnage central de l'histoire s'accompagne, notamment sous la Monarchie de Juillet, d'une nouvelle rationalité scientifique. Le peuple est décrit dans ses caractères physiologiques, constituant un langage naturel dont l'historien anthropologue doit découvrir la signification. Dès lors, on proposerait un autre sens au terme de biographie, exprimant cette modification des recherches historiques. Le terme ne s'entendrait plus simplement dans son sens habituel, c'est-à-dire le récit, l'écriture de la vie d'un individu ou d'un peuple. Il revêtirait une autre acception: la biographie ne serait pas seulement écriture, mise en discours de la vie, mais celle-ci constituerait, d'elle-même et immédiatement, un discours, une expression de la nature. La «bio-graphie » ne serait plus alors l'écriture de la vie, mais la vie déjà langage, une écriture vivante, une graphie biologique, une parole naturelle et incarnée : le langage même de la nature. Il n'y aurait pas d'anthropologie sans bio-graphie, c'est-à-dire pas de grille de lecture des indices naturels de l'humanité sans, corrélativement, un langage vivant des corps à déchiffrer. C'est ainsi que chez l'abbé Frère, Alphonse Esquiros et les anthropologues les crânes s'offrent comme une inscription hiéroglyphique, "bio-graphique » à partir de laquelle il devient possible de reconstituer la biographie d'un être. La transformation historiographique correspondrait par conséquent au passage politique d'une histoire pensée comme récit des actions, exploits et propos mémorables des grands hommes, à une histoire relatant la destinée des nations. L'évolution se manifesterait, de plus, dans la substitution scientifique d'une histoire conçue comme écriture biographique de la vie des grands personnages de l'Etat, au déchiffrement "bio-graphique» des signes physiques des races, constituant une véritable langue naturelle des corps. L'histoire décrit alors la Vie, dans son sens le plus matériel et le plus biologique, des peuples. La pensée accoste à ce moment sur un nouveau continent historique. L'histoire des sociétés se trouve inscrite dans l'histoire des corps. On écrit la biographie des peuples dans la bio-graphie de leurs crânes. Désormais, pour décrire le progrès, il importe d'interpréter le "grand livre de la vie $»^{50}:$ il faut observer pour lire, et lire pour écrire l'histoire. Le langage de l'historien se veut une traduction de cette voix naturelle des corps dans l'histoire: le Corpus de l'histoire. Avec l'abbé Frère et Esquiros, l'historien devient le Champollion des nations dans l'histoire.

22 L'histoire, envisagée par Alphonse Esquiros, suit donc un plan naturel, universel et divin. Elle est marquée par le Progrès et la répétition, du macrocosme au microcosme, de la même loi gouvernant ses phénomènes. Après les deux cercles du progrès naturel, celui de la cosmologie de l'histoire et de la science des races, puis celui du perfectionnement de chaque race décrit par l'abbé Frère, fragment de la perfectibilité universelle, apparaît un dernier cercle, celui du progrès naturel des races dans l'histoire de France, culminant dans l'avènement de la Seconde République. 


\section{La dialectique du progrès dans l'histoire de France}

\section{Le socle des races de l'histoire de France}

23

Pour Esquiros, les secrets de l'histoire de France résident dans l'organisation des peuples qui se sont trouvés réunis, par le fracas des conquêtes, sur le sol de la Gaule. La clef de l'histoire nationale, les transformations de la société, les mouvements et les progrès politiques de l'Etat trouvent leur racine dans le socle des races qui en dessine la trame. L'histoire de France est inscrite en filigrane dans les caractères des nations qui s'y combattent : «Si le secret des nationalités repose sur le secret de l'organisation des races, si ces races elles-mêmes ne sont que les notes d'un vaste concert que la civilisation arrange, modifie, perfectionne incessamment, on voit d'ici quelle révolution le $\mathrm{xIx}^{\mathrm{e}}$ siècle est appelé à introduire dans l'histoire $~^{51}$. Là réside le principe d'intelligibilité, nouveau et irremplaçable, de l'histoire de France. Il faut donc introduire l'ethnologie dans la philosophie politique : c'est la nature des races qui détermine la forme de l'organisation sociale.

24 A la suite des historiens bourgeois, Augustin Thierry (1795-1856) et son frère Amédée (1797-1873), François Guizot (1787-1874), etc., Alphonse Esquiros cherche à explorer les fondements de l'histoire de France dans les antipathies des populations qui, sous les institutions et les lois, à travers les vicissitudes de la politique, expliquent son évolution et son sens. En effet, la nation française est sortie de l'association, de l'amalgame violent de trois races: la race celtique, formant la couche primitive de la population, la race romaine et la race des Francs. Esquiros part du schéma élaboré par Augustin Thierry et F. Guizot pour mettre en lumière la dynamique cachée de l'histoire du pays.

- La population celte de la Gaule est asservie, au premier siècle avant notre ère, par la race romaine qui occupe son territoire. Ces deux races fusionnent, disent les historiens bourgeois, pour constituer une seule entité, harmonieuse et unie : la race gallo-romaine.

- $A u v^{e}$ siècle, la race des Francs soumet la nation gallo-romaine, imposant sa domination à travers les lois et les institutions. Cette lutte de races se perpétue, après la victoire, à travers la société : les nobles seigneurs, progéniture des Francs victorieux, asservissant le tiers état, issu des Gallo- romains vaincus ${ }^{52}$. Augustin Thierry produit ainsi à partir de cette haine des races sa «théorie de la dualité nationale " ${ }^{53}$.

- L'antagonisme des nations, mêlées de force sur le sol du même pays, prend alors l'allure d'une lutte de classes. Augustin Thierry met en forme le modèle historiographique de cette transformation d'une guerre de races étrangères en lutte de classes, constitutive de la structure sociale des États. L'étude historique des races sous-tend une sociologie des classes. Esquiros le soutient à son tour, les peuples ennemis ont institué, sur le sol national, des classes adverses : « Les classes ont commencé par être des races $»^{54}$.

- La Révolution de 1789 marque le renversement du rapport de force en faveur de la roture contre les héritiers de leurs oppresseurs francs ${ }^{55}$. Le tiers renverse la monarchie et la noblesse, annulant ainsi les effets politiques et sociaux de la conquête. La Révolution s'offre par conséquent comme une reconquête, une revanche des anciens vaincus sur leurs vainqueurs d'hier.

L'Émpire continua l'œuvre de fraternisation amorcée par la Révolution. L'entreprise reçoit un coup d'arrêt avec la Restauration, ce qui déclenche le combat théorique engagé par Guizot et Augustin Thierry contre ce régime. Cette mission est reprise et achevée par

Revue d'histoire du XIXe siècle, 20/21 | 2000 
la Monarchie de Juillet qui, en instaurant un régime libéral, garantissant l'égalité juridique de tous les citoyens, abolit pour toujours, pensent ces historiens, la haine des races et des classes qui divisait la nation française. Il n'y a plus alors ni oppresseurs ni opprimés, tous les citoyens sont libres. L'ère des révolutions est révolue. Augustin Thierry prophétise alors une sorte de fin de l'histoire. La révolution de 1848 se chargea de réfuter ses prédictions.

\section{La politique de l'histoire et la réfutation de l'histoire bourgeoise}

La pensée d'Augustin Thierry noue, de manière frappante, la politique et l'histoire, la réalité présente et l'interprétation du passé. Il met en forme une politique de l'histoire. C'est la Révolution politique, marquant l'irruption des masses sur le théâtre de l'histoire, qui introduit une révolution scientifique dans la manière d'écrire l'histoire. Esquiros ne dit pas autre chose: "L'histoire doit participer aux changements qui se font dans l'ordre politique $\aleph^{56}$. Le présent trouve son sens, sa rationalité et sa vérité dans l'exploration du passé de la France. La conquête franque du $\mathrm{v}^{\mathrm{e}}$ siècle rend raison des clivages politiques contemporains. Ainsi, le passé détermine l'actualité. Mais, inversement, la connaissance authentique de l'histoire tire du présent, des événements politiques, c'est-à-dire de la Révolution, son intelligibilité. C'est à la lumière de la crise révolutionnaire que le mouve ment secret et profond de l'histoire de France se manifeste dans toute sa clarté.

Mais si le présent délivre la vérité du passé, toutes les modifications du présent bouleversent la connaissance que l'on peut avoir de l'histoire. Les révolutions actuelles modifient le sens des révolutions passées. La politique en acte répudie la connaissance historique, le passé est rattrapé par le présent. L'histoire se faisant réfute l'histoire des savants. En effet, dans la théorie d'Augustin Thierry la révolution de 1848 est impossible car impensable dans une société réunifiée. Son éclatement a donc eu pour effet de mettre à mal l'analyse historique qui était proposée de l'histoire nationale. Il n'a pas, au demeurant, dissimulé son désarroi. L'événement a anéanti toute la connaissance qu'il prétendait avoir du passé57.

Alphonse Esquiros écrit Les fastes populaires après la révolution de 1848 pour magnifier le progrès millénaire du peuple qui émerge dans ce grand événement. S'il s'inscrit dans le sillage des historiens bourgeois, aux constructions historiographiques récusées, il recueille leur héritage et propose, à partir de leur théorie, un nouveau canevas historique susceptible de rendre raison de l'actualité. Si toute révolution doit se penser comme une revanche ou une reconquête d'une race jadis asservie, rappeler, dans l'analyse historique, une conquête c'est, ipso facto, trouver la racine d'une révolution passée ou à venir. De ce fait, Esquiros, partant du modèle proposé par Augustin Thierry, le complète et l'achève en rappelant la conquête des Romains qui, malgré la conquête franque, n'a pas annulé ses effets. Ainsi, l'invasion romaine a-t-elle institué la domination de la race romaine sur la nation celte, l'irruption franque ayant imposé le pouvoir de cette race sur ces deux populations. Par conséquent, ces trois races ont constitué trois classes. La race gauloise est devenue la classe populaire et laborieuse des villes et des campagnes, la race romaine a donné la classe bourgeoise : « L'opinion qui fait descendre la classe moyenne, en France, du sang romain dégénéré, est une opinion neuve, qui rencontrera, nous n'en doutons point, des incrédules ${ }^{58}$. La race des Francs a constitué la classe noble. La hiérarchie des races a débouché sur une hiérarchie des classes. Dans ce dispositif racial et social, les peuples gaulois et romain n'ont pas fusionné, comme le pensent Augustin Thierry et 
Guizot, ils ont conservé leur antagonisme de races et leur hostilité de classes. Il n'y a jamais eu de race gallo-romaine, bien qu'Esquiros utilise parfois l'expression. La race gauloise a subsisté après l'invasion dans toute son intégrité : «Il y aurait donc de la naïveté à penser avec Claude, et avec les historiens français qui l'ont cru sur parole, que l'invasion romaine ait enlevé à la race celtique ses caractères ${ }^{59}$. Aussi, la Révolution de 1789, confortée par celle de 1830, a-t-elle bien donné lieu à un affranchissement, mais pour la race romaine uniquement : elle fut une révolution de la bourgeoisie. Si elle annula la conquête des Francs, en émancipant les Romains, elle a maintenu dans la servitude la race gauloise, qui attendait son heure pour secouer le joug de la domination bourgeoise. La révolution de 1848 s'offre alors, dans cette perspective, comme l'émancipation de la classe ouvrière, descendant des Gaulois, venant se libérer de l'exploitation bourgeoise, c'est-à-dire romaine : «Il fallut des siècles pour que la bourgeoisie rentrât pied à pied dans la propriété de la terre dont elle avait été violemment expulsée par la conquête ; ce fut une œuvre lente, mais continue, mais imperturbable. Cette œuvre était déjà fort avancée en 89 ; la classe moyenne avait mis la main sur la plus grande partie des biens que la race conquérante lui avait arrachés. La révolution acheva de déposséder les anciens envahisseurs. Au point de vue où la bourgeoisie révolutionnaire se plaçait vis-àvis de la noblesse, la vente des biens nationaux ne fut pas autre chose qu'une restitution. Ce qui étonne, c'est qu'au moment où la classe moyenne croyait rentrer dans son droit, en rentrant dans les terres dont ses aïeux avaient été chassés par les ancêtres des familles nobles, elle n'ait pas songé à ces plus anciens propriétaires du sol, aux Gaulois que l'invasion romaine avait antérieurement dépouillés. Ils étaient pourtant sous ses yeux, ces descendants de la race celtique, aujourd'hui les paysans de nos campagnes et les ouvriers de nos villes! Ils étaient là, marqués dans leur dégradation et leur misère par le stigmate de la défaite. - La justice voulait que dans ces jours de réparation, dans ce grand jubilé social de 92, on les fit rentrer, eux aussi en possession de leurs biens. Si la bourgeoisie eût compris cela; si elle eût associé l'autre classe aux conséquences de sa victoire, elle eût terminé alors l'ère des révolutions ; car l'œuvre du progrès national est d'effacer dans la société la trace toujours subsistante de la conquête ${ }^{60}$.

Premier occupant du sol, le peuple gaulois est le dernier à subir les conséquences des conquêtes romaine et franque. Il reste donc à décrire par quel mécanisme Esquiros explique sa lente accession à la liberté. C'est dans l'organisation physique et morale de cette race, mère des classes laborieuses de la France, qu'il faut chercher son progrès vers l'émancipation, consacrée par la Seconde République.

\section{La race Christ et l'Eucharistie de l'histoire}

30 La race gauloise constitue la "race mère ${ }^{61}$ de la nation française. Les celtes se caractérisent par des qualités éminentes: l'initiative, l'action, le désintéressement, le besoin organique d'élever à soi tout ce qui est inférieur et souffre. Amoureuse de la liberté, cette ethnie porte également un grand respect à la personne des femmes. La race primitive du sol national se couvre d'une dimension mystique sous la plume gallocentriste d'Esquiros. Son dévouement, son sens du sacrifice l'invite à communiquer à tous les autres peuples ses vertus. Elle est vouée au partage, à l'image d'une race Christ: «Parmi les familles humaines, il en est qui reçoivent et d'autres qui communiquent. La race celtique est de ces dernières : elle reçoit peu; elle communique toujours. $\mathrm{Si}$, comme nous le croyons, la véritable grandeur se mesure au sacrifice, la race celtique est la première des races vivantes. Toujours et partout, dans les temps plus reculés, comme aux 
époques modernes, nous trouvons la nation gauloise indomptable dans sa générosité, versant sa lumière et ses souffrances sur les autres peuples; nous la voyons, race sacrée, race martyre, race Christ, convier toutes les nations de la terre à une sorte de communion perpétuelle : Mangez, leur dit-elle, voici ma chair ! Buvez, leur dit-elle, voici mon sang ! Et par la puissance, du sacrifice, elle vivifie en elle toute l'humanité $»^{62}$.

31 La nation gauloise se trouve donc investie d'une mission religieuse et sacrée : porter au monde le message de la liberté et de la fraternité. Elle est animée d'un mouvement d'expansion, d'une force centrifuge qui ramènent tous les peuples dans son sillage. C'est pourquoi, si l'humanité se compose de races mâles et de races femelles, elle tient à la fois des deux sexes, étant ainsi par sa nature, l'agent médiateur, le peuple intercesseur, la race faite de toutes les races du globe : « Il y a, si l'on ose ainsi dire, dans l'humanité, des races mâles et des races femelles. La race gauloise ou celtique participe à la fois du caractère moral des deux sexes. De là sa supériorité, de là cette alliance de l'idée et de l'action, de l'inspiration et de la science, de la rêverie et de la force, qui distingue la nation française " ${ }^{63}$. Décrire la force d'expansion de la race gauloise dans l'histoire permet d'expliquer comment elle a pu, peu à peu, absorber en elle les races conquérantes romaine et franque pour s'affranchir de leur domination, dynamique culminant dans la révolution de 1848.

Alphonse Esquiros décrit trois énergies d'expansion du peuple gaulois permettant de rendre raison de son essor et de son affranchissement. Il s'agit, d'abord, de la dynamique intrinsèque du progrès inscrite dans le cerveau des races, selon les thèses soutenues par l'abbé Frère. Ensuite, le travail, l'activité économique est, aux yeux de notre auteur, une force pacifique d'émancipation. L'histoire des Gaulois devient l'histoire du travail, du prolétariat et de son essor vers la liberté. La dernière source d'explication du progrès de la race gauloise réside dans l'hybridité. Celle-ci constitue la puissance principale du perfectionnement des peuples : " - De toutes les causes qui concourent à effacer dans une nation les inégalités de races, sur lesquelles se fondent les inégalités de condition entre les hommes, la première et la plus active est, sans contredit, le croisement ; [...] ${ }^{64}$. C'est dans le métissage que se révèlent les arcanes de l'histoire de France. Ce mouvement biologique et mystique, cette œuvre indistinctement religieuse et naturelle, brosse un tableau nouveau de l'histoire de France. Si c'est par la communication et le croisement que s'opère le progrès des races, l'histoire se pense sous les traits d'une grande Eucharistie, d'une vaste communion dirigée par la race Christ, versant sa vie dans tous les sangs étrangers : "La société est un banquet: nous nous donnons les uns aux autres, et loin de rien perdre à ce partage, nous y gagnons de ressembler de plus en plus à la Divinité qui donne et qui donne toujours. Nous passons en quelque sorte notre chair et notre sang aux autres sans cesser pour cela d'être nous-mêmes; car les générations futures avec lesquelles nous sommes d'avance en rapport, participeront un jour aux progrès que nous avons, pour ainsi dire, marqués dans leurs organes. C'est le grand mystère de la communion historique ${ }^{65}$.

33 L'histoire de France n'est qu'un lent et continu processus d'assimilation des races diverses et ennemies qui s'affrontent sur son territoire. La nation gauloise assume cette mission d'unification de la société en s'amalgamant les ethnies hétérogènes qui s'y trouvent mêlées par le feu des invasions : «Le travail de la civilisation est d'amalgamer ces éléments hétérogènes $»^{66}$. Ce mouvement s'opère à la fois vers le haut et vers le bas. La race celte s'est en effet liée aux peuples proscrits et méprisés : cagots, juifs, bohémiens, restés en marge de la société française. Elle les a assimilés à sa personne. Dans cette 
œuvre de synthèse historique, elle recueille en elle leurs souffrances, leurs révoltes et leurs espoirs.

Le perfectionnement social résulte directement de cette dynamique du mélange des races hétérogènes. Elles obéissent à un plan divin et naturel dictant le progrès humain : «Les instincts de race, en apparence les plus aveugles, servent, nous le croyons, des intentions et des vues supérieures, cachées pour ainsi dire dans les organes de la vie par la main de la Providence ${ }^{67}$. Le progrès des races, par une sorte d'hybridité sacrée, devient dès lors inéluctable et nécessaire : il constitue une loi de la nature et de Dieu. Chaque peuple a versé, à son heure, sa contribution au creuset de la race gauloise pour la vivifier et la fortifier : «Les races, dans leurs caractères primitifs comme dans leurs transformations successives, sont, pour ainsi dire, les dépositaires des matériaux dans lesquels puise la main de la Providence pour augmenter la vie intellectuelle et morale des peuples $»^{68}$.

Mais elle s'est également mélangée, vers le haut si on peut dire, avec les races romaine et franque qui l'avaient asservie. Le processus historique qu'il décrit prend alors la forme d'une authentique dialectique. Le croisement constitue, comme chez Victor Courtet de l'Isle, un véritable moteur dans l'évolution des sociétés ${ }^{69}$. C'est par ce mécanisme que le progrès se communique d'une race à l'autre, et donc d'une classe à une autre en tendant vers l'unité.

\section{Dialectique libératrice du mélange des races}

La nature a imposé des règles strictes dans le mélange des races, souhaitant constamment que ces unions engendrent un progrès : « La nature veut l'élévation des races, elle ne veut pas leur abaissement $»^{70}$. Elle a donc prescrit que le croisement s'opère entre un homme d'une race supérieure et une femme d'une race moins favorisée: "Suivez d'ailleurs de près la voie par laquelle les races tendent vers l'unité : vous verrez que c'est toujours l'homme d'une race supérieure qui descend vers la femme d'une race inférieure ${ }^{71}$. Il évoque les travaux anthropologiques d'Etienne Serres pour montrer que la reproduction d'un homme blanc avec une femme noire est plus favorable que celle d'une femme blanche avec un homme noir ${ }^{72}$. Esquiros applique ce principe physiologique à l'histoire du pays. Toutes les races souhaitent en effet s'assimiler les autres par le croisement : «Les races tendent à l'envahissement; toutes veulent marquer leur empreinte sur les autres races $»^{73}$. C'est ainsi qu'il montre les effets sociaux et politiques du droit de culage et de jambage, exercé par la race des Francs sur les races gauloise et romaine. Ce privilège des oppresseurs était pensé comme la conséquence ultime de la victoire: le peuple conquérant n'avait pas seulement asservi les populations, fait main basse sur les terres, il voulait coloniser les berceaux en imposant à la descendance celte les traits de sa race. Ce droit inique, emblème même de l'oppression, devait servir une fin exactement contraire. En effet, les conquérants francs, jaloux de leur supériorité, refusaient toute union légale avec la population vaincue. Cet orgueil constituait un obstacle insurmontable à la réalisation des vœux de la nature, souhaitant l'unité par la fusion des peuples. Mais paradoxalement, c'est par le vice que les dominateurs exaucèrent le vœu de la nature qu'ils refusaient d'accomplir par le droit. Le droit de culage et de jambage eut pour effet d'égaliser les races franque et gauloise : «Le droit de culage a contribué à verser du sang noble dans le sang plébéien; il a donc été sous ce rapport le moyen matériel dont s'est servi la nature pour égaliser les classes en mêlant les races ${ }^{74}$. L'égalité biologique entre les races, née du croisement, introduit une nécessaire égalité sociale, apportée par la 
Révolution. Celle-ci apparait moins comme un événement politique que comme la conclusion anthropologique d'un abus de droit. Esquiros explique : "Pour arriver à ses fins, la nature appela à son secours cet instinct charnel, ce besoin de communication brutale, qui porte le tempérament mâle de la race conquérante sur les filles des races conquises. Qu'arriva-t-il ? C'est qu'avec le temps, les hommes de l'aristocratie transmirent par le droit de culage leurs caractères naturels à la famille de leurs vassaux. Aussi bien il se rencontra un jour où toute la nation eut du sang noble dans les veines. Ce jour-là le peuple tout entier se leva, se proclama lui-même le Souverain $»^{75}$.

37 Il conçoit de ce fait la Révolution française à partir du métissage. Il propose en cela une authentique dialectique naturelle pour expliquer l'histoire de France. Le croisement des races assume une fonction religieuse en réconciliant des races diverses et ennemies, il les re-lie, les associe pour les réconcilier selon le décret de la Providence, souhaitant l'unité des races. Il surmonte leur antagonisme pour les unir dans une totalité et une synthèse supérieures. Il explique le croisement entre la race des Francs et celles des Gaulois et des Romains par une sorte de ruse de la raison de type hégélien. L'injustice est l'instrument même par lequel se réalise la justice. La violence et l'usurpation des conquérants, portées à leur point le plus haut par le droit de jambage, réalisent leur contraire : la justice et l'égalité. Le signe le plus éclatant de la conquête militaire consacre la défaite de la race des vainqueurs : "Il est impossible de ne point admirer ici le secret dessein de la justice divine qui a mis la punition dans le désordre même, et qui a voulu que le fort se blessât à ses propres armes. Elle a dit à la noblesse : Tu tomberas par où tu as péché $! »^{76}$. La justice vient de l'injustice, la liberté de la domination, la force s'effondre par le viol de la faiblesse, l'emblème de l'inégalité contribue à l'égalité, le désordre produit l'ordre : « En histoire, le mal est un bien dont nous ne saisissons pas les rapports [...] $\gg^{77}$. Dans cette ruse naturelle de l'histoire, tout contribue au progrès des hommes et des sociétés, dans un plan naturel voulu par Dieu, même ce qui, au premier regard, semble accomplir le contraire. Il le proclame : «Il y a donc une loi dans la nature qui fait servir l'injustice des hommes à la justice et au progrès des sociétés. Au vol de la terre, le crime qui succède après les grandes invasions, c'est le viol de la race conquise : quand on songe que ce crime a duré des siècles, et qu'il s'est éteint dans sa propre honte, on se demande où était Dieu ?... Derrière cet outrage fait au droit des gens, il y a pourtant une loi de la nature qui agit et qui se sert, pour agir, des mauvais instincts du cœur de l'homme. En cédant à ses convoitises brutales et en humiliant sous son libertinage les races vaincues, la race victorieuse tend à s'abdiquer elle- même. La Providence a caché son châtiment dans le plaisir, surtout quand ce plaisir est injuste : elle a voulu que les nations conquérantes, en abusant des nations conquises, leur transmissent, par voie de génération, ces caractères de race dont elles sont si jalouses. Ce mouvement est lent sans doute; mais infaillible. Les forts se dépossèdent eux-mêmes en violant le droit des faibles dans ce que ces droits ont de plus délicat et de plus sacré, l'honneur de la femme $»^{78}$.

La Révolution de 1789 a donc secoué le joug de la souveraineté franque en renversant le pouvoir de la classe noble et de la monarchie. Mais elle n'acheva pas pour autant d'émanciper le peuple celte. Si les populations gauloise et romaine, devenues le prolétariat et la bourgeoisie, se débarrassèrent de leurs envahisseurs d'alors, la classe ouvrière subissait toujours la domination politique et l'exploitation économique de la classe moyenne : «La noblesse a pesé sur la bourgeoisie : la bourgeoisie pèse, depuis 89 , sur l'ouvrier des villes et des campagnes $»^{79}$. L'ère des insurrections n'était pas close : « La civilisation continue de vivre sur les suites d'une guerre sociale $[\ldots] »^{80}$. La lutte des 
classes et des races continuait, en dépit des prophéties d'Augustin Thierry, comme l'atteste la révolution de 1848 , étape essentielle du mouvement millénaire de libération du peuple. Et de même que le droit de jambage ruina le pouvoir de la race des Francs sur les races asservies, ce viol exercé maintenant par la classe bourgeoise dans les manufactures produira les mêmes effets sociaux et politiques : «La noblesse, par l'abus d'un droit inique, a fini par perdre tous ses autres droits. Il en sera de même dans la suite pour la bourgeoisie, si la classe imite les déportements de l'aristocratie ${ }^{81}$. En effet, l'abus de ce droit entraînera nécessairement l'égalisation organique des races, donc leur égalité sociale : « Les excursions de la classe bourgeoise sur le terrain des classes populaires, pour $\mathrm{y}$ chasser aux jeunes filles, auraient absolument les mêmes résultats que le privilège exercé par les seigneurs sur les vassales. La possession, ou pour mieux dire, l'usurpation de la femme, qui est, en elle-même, le plus monstrueux des crimes, a des suites qui tendent, comme nous l'avons vu, à l'unité de race, c'est-à-dire à l'égalité des citoyens et à l'effacement des classes ${ }^{82}$.

Dans la philosophie de l'histoire exposée par Esquiros, les exclus, les marginaux, les sans voix de l'histoire traditionnelle retrouvent leur dignité. Ainsi la race gauloise devient-elle le personnage central de la scène historique, les races proscrites : les cagots, les juifs, les bohémiens se trouvent intégrées dans le schéma d'analyse de l'histoire. Elles y retrouvent leur juste place, mettant fin à l'injuste anathème dont elles étaient victimes, en apportant leurs qualités au creuset gaulois. Enfin et surtout, par le biais d'une réflexion physiologique et historique sur les croisements, la femme retrouve sa place nodale dans le progrès des sociétés. Elle constitue la matrice assimilant les races étrangères au peuple celte. C'est la nature de la femme qui lui assigne une fonction majeure dans un processus historique déterminé par des lois anthropologiques. Elle enfante l'association des races et l'unité sociale: « La race celtique n'en est pas moins une race puissante et indestructible qui, à cause de ses affinités avec le sol, tend toujours à reparaître sous les deux autres races étrangères. Nous croyons qu'elle finira par les absorber et par leur donner sa figure. La femme, au moyen des secrètes attractions de sa nature, se montre, ici comme partout, le terrain vivant, pour ainsi dire, de l'assimilation des races, le moule mystérieux du progrès, moule à travers lequel les races dans le genre humain, les classes dans l'ordre social, arriveront à l'unité $»^{83}$.

Ce mouvement naturel d'assimilation des races, dont les femmes sont le pivot, s'étend audelà des frontières de la France : «Si cette remarque pouvait s'étendre aux autres races, la femme, agent actif dans l'œuvre de la reproduction, se montrerait à nous comme le moule du progrès ; or la science entrevoit déjà la certitude d'élever ce fait à la hauteur d'une loi générale $»^{84}$. Esquiros pense en effet que la race gauloise est investie, par la nature et la Providence, d'une mission universelle. Elle doit unifier l'Europe et le monde en s'appuyant sur les embryons de voies de communication en construction (voies ferrées, lignes maritimes, canaux ${ }^{85}$. C'est alors que la philosophie de l'histoire de France fait retour vers l'universel.

41 Alphonse Esquiros a donc voulu poser les bases d'une nouvelle philosophie de l'histoire en l'inscrivant dans le mouvement universel de la nature. Il faut sans doute cesser de penser, rétrospectivement, que l'invocation des races n'a joué, dans le discours historique et politique, qu'une fonction de légitimation de l'asservissement de races que l'anthropologie $\mathrm{du} \mathrm{XIX}^{\mathrm{e}}$ siècle aurait, délibérément, infériorisées pour justifier son européocentrisme. La notion, au siècle passé, s'est chargée d'une profonde dimension démocratique: elle a permis de montrer le rôle nodal du peuple et des masses dans 
l'histoire et d'expliquer le perfectionnement des sociétés. Enfin, dans cette synthèse encyclopédique, Esquiros mobilise l'ensemble des sciences naturelles de son temps pour expliquer le processus de développement du peuple dans l'histoire nationale, abrégé du progrès universel de la nature. Le socialisme se trouve, dans son œuvre, doté de fondements naturalistes et scientifiques. Sa pensée invite à questionner le statut même $\mathrm{du}$ discours socialiste en interrogeant les rapports entre l'utopie et la science, entre le socialisme et le savoir. En refusant de considérer rétrospectivement le socialisme comme une pure chimère - qu'il faudrait « dissiper à la brume de la raison enfin montée à son zénith $»^{86}$ dirait Foucault - on pourrait, à la suite d'Esquiros, le réinscrire dans l'épistémè de son temps. Par ce biais, il deviendrait alors possible de comprendre comment et pourquoi il prétendait accéder à l'universalité objective de la Science. Bonnet-Duverdier, à la même époque, exprimait sans détour cette prétention: "Qu'est-ce à dire et que prétendons- nous? Une chose très simple et incontestable, à savoir: que l'heure est venue de donner aux choses leur vrai nom, et que cette heure ne pouvait sonner plus tôt ; que ce que nous appelions, hier encore, d'un nom mystique, doit s'appeler aujourd'hui d'un nom plus clair ; que le sentiment a fait place à la connaissance dont il n'était que l'intuition; qu'en un mot le socialisme, en 1854, doit revêtir un autre nom et s'appeler purement et simplement la science $»^{87}$.

\section{NOTES}

1. Alphonse ESQUIROS, "De l'Assemblée Nationale ", L'Artiste. Revue de Paris. Beaux-arts et Belles lettres, $\mathrm{V}^{\mathrm{e}}$ série, Tome I, $2^{\mathrm{e}}$ livraison du 26 mars 1848, p. 84.

2. Idem, p. 84.

3. Sur Alphonse Esquiros, voir Jacques P. VAN DER LINDEN, Alphonse Esquiros, de la bohème romantique à la république sociale, Paris, A.-G. Nizet, 1948, 237 p.; Anthony ZIELONKA, Alphonse Esquiros (1812-1876) : a study of his works, Paris/Genève, Champion/Slatkine, 1985, 300 p. Voir aussi sur l'ensemble des questions traitées dans ce texte, Claude BLANCKAERT, «Les Gaulois au Muséum: savoirs naturalistes et principe des nationalités à l'époque romantique ", Revue d'histoire des sciences, 1998, tome 51, no 4, pp. 457-505.

4. En fait, le livre s'arrête au seuil $\mathrm{xVII}^{\mathrm{e}}$ siècle, il devait être suivi par une Histoire de la démocratie moderne qui n'a pas été publiée. Il faut noter qu'en tant que représentant du peuple, il dut s'exiler après le coup d'État du 2 décembre. Les Fastes populaires permettent néanmoins de saisir la logique d'ensemble de la pensée historique d'Esquiros.

5. Alphonse ESQUIROS, Les Fastes populaires ou Histoire des actes héroïques du peuple et de son influence sur les sciences, les arts, l'industrie et l'agriculture, tome premier, Paris, Administration des publications populaires, 1851, Introduction, p. CXXXVII.

6. Alphonse ESQUIROS, Paris ou Les sciences, les institutions et les mœurs, tome I, Paris, au Comptoir des imprimeurs unis, 1847, Introduction, p. 7.

7. Alphonse ESQUIROS, Les Fastes populaires..., tome deuxième, Paris, 1852, p. 43.

8. Alphonse ESQUIROS, Paris..., ouv. cit., Introduction, p. 6.

9. Alphonse ESQUIROS, Les fastes populaires..., tome troisième, Paris, 1852, p. 3.

10. Alphonse ESQUIROS, Charlotte Corday, 1840, dans Les mille et un romans, nouvelles et feuilletons. Tome dix-septième, Paris, Boulé, 1846, p. 30. 
11. Alphonse ESQUIROS, De la vie future au point de vue socialiste, Paris, 1850, Librairie phalanstérienne, p. 84.

12. Alphonse ESQUIROS, Histoire des Montagnards, 1847, dans Veillées littéraires illustrées. Choix de romans, nouvelles, poésies, pièces de théâtre, etc., etc. Par les meilleurs écrivains anciens et modernes, Tome V., Paris, J. Bry aîné, 1851, p. 71.

13. Alphonse ESQUIROS, Les Fastes populaires..., tome deuxième, Paris, 1852, p. 2.

14. Alphonse ESQUIROS, Paris..., ouv. cit., Introduction, p. 2.

15. Alphonse ESQUIROS, Les Fastes populaires..., tome deuxième, Paris, 1852, pp. 13-14.

16. William-Frédéric EDWARDS, «Esquisse de l'état actuel de l'anthropologie ou de l'histoire naturelle de l'homme ", Mémoires de la Société ethnologique, tome premier, deuxième partie, Paris, 1841, p. 109.

17. Alphonse ESQUIROS, Les Fastes populaires..., tome deuxième, Paris, 1852, p. 2.

18. Le saint-simonien Gustave d'Eichthal (1804-1886), secrétaire de la Société ethnologique de Paris, l'explique remarquablement dans un manuscrit: "La Genèse nous dit, qu'au commencement Dieu créa chaque chose suivant son espèce; Les peuples aussi ont été créés chacun suivant son espèce; et les espèces sont impérissables. Certes, si une époque a eu abondamment la preuve de cette vérité, c'est bien la nôtre. La terre est aujourd'hui comme la vallée de Josaphat au dernier jour; Ce ne sont que Peuples sortant de leur Cercueil; si le siècle dernier a été le siècle de l'insurrection le nôtre pourra s'appeler celui de la résurrection. La race Gallique s'est conservée en Écosse, en Irlande, dans le pays de Galles, en Bretagne, et réclame sa nationalité. La Belgique a conquis son indépendance, et en France même les diverses variétés provinciales se réveillent. Les différentes peuplades de l'Espagne secouent le joug sous lequel on a voulu les confondre ». Gustave d'EIGHTH AL, Extrait d'une lettre à $M^{r}$.... à Paris, envoyée de Vienne le 15 janvier 1837, Bibliothèque de l'Arsenal, Fonds Enfantin, [Ms.7825/15, deuxième feuillet ] Souligné par l'auteur.

19. Victor Courtet de l'Isle (1813-1867) explique, en 1849, que le Printemps des peuples est un enfantement des races : «Or, surtout dans ces temps d'ébranlement politique, lorsque l'Europe continentale est en feu, lorsque le monde est dans l'enfantement, qu'on examine le mobile de toutes ces agitations: on verra, sur presque tous les points, que le combat est engagé sur une question de races ». Victor COURTET de l'ISLE, Tableau ethnographique du genre humain, Paris, Arthus Bertand, 1849, Avant-propos, non paginé.

20. Euryale CAZEAUX, «Lettre sur le programme à suivre par la Société ethnologique, Paris, le 20 mai 1839 », lue à la séance du 27 mars 1846, Bulletin de la Société ethnologique de Paris, tome premier, 1846, Paris, 1847, p. 20.

21. Alphonse ESQUIROS, Les Fastes populaires..., tome premier, Paris, 1851, Introduction, p. II.

22. Alexandre Moreau de Jonnès (1776-1870) explique en ce sens : «L'origine des peuples peut expliquer presque toujours les mystères de leur histoire ; et il suffit de l'indication de la race dont ils descendent, pour révéler le degré de leur habileté militaire, leur inclination pour la culture des arts, leur aptitude à la civilisation, leur supériorité intellectuelle et morale, leur résignation à porter le joug de l'esclavage ou leur résistance à l'envahissement de leurs libertés ». Alexandre MOREAU de JONNÈS, Statistique des peuples de l'Antiquité. Les Égyptiens, les Hébreux, les Grecs, les Romains et les Gaulois. Économie sociale, civile et domestique de ces peuples, Paris, Guillaumin et $C^{\text {ie }}$ 1851, pp. 578-579.

23. Émile MONTÉGUT, «Du génie de la race anglo-saxonne et de ses destinées », Revue des deux mondes, XXI ${ }^{\mathrm{e}}$ année, Nouvelle période, 1851, tome III, livraison du 15 septembre 1851, p. 1028.

24. Alphonse ESQUIROS, Les Fastes populaires..., tome deuxième, Paris, 1852, pp. 2-3.

25. Il faut préciser que je ne prétends pas ici commenter en tant que telles les thèses anthropologico-historiques de l'abbé Frère, mais la lecture, partielle et partiale, qu'Alphonse Esquiros en fait en les pliant à ses propres vues.

26. Idem, p. 12.

Revue d'histoire du XIXe siècle, 20/21 | 2000 
27. Idem, p. 21.

28. Il existe en fait huit périodes pour l'abbé Frère, il qualifie la dernière de période stationnaire. 29. Alphonse ESQUIROS, "Des études contemporaines sur l'histoire des races", Revue des deux mondes, tome vingt-unième, dix-huitième année, nouvelle série, janvier-mars 1848 , livraison du 15 mars 1848, p. 989.

30. L'abbé Frère écrit: «Nous croyons qu'il existe un développement gradué dans les crânes depuis la première période jusqu'à la dernière ; que la forme, le dessin, les proportions entre la partie postérieure et antérieure, entre le crâne et la face, changent avec la période, et vont toujours en augmentant et en s'harmonisant ». Abbé Ph.-A. FRÈRE, Principes de la philosophie de l'Histoire, Paris, Gaume frères, 1838, note (1) des pp. 173-177 et p. 174.

31. Alphonse ESQUIROS, « Des études contemporaines sur l'histoire des races », art. cit., p. 988.

32. Alphonse ESQUIROS, Les Fastes populaires..., tome deuxième, Paris, 1852, p. 9.

33. Alphonse ESQUIROS, «Le docteur Gall », Revue de Paris, quatrième série. Année 1843, tome quatorzième, livraison du 5 février 1843, p. 40.

34. Alphonse ESQUIROS, Les Fastes populaires..., tome deuxième, Paris, 1852, p. 10.

35. Idem, p. 10.

36. Alphonse ESQUIROS, Paris..., ouv. cit., pp. 337-338.

37. Idem, p. 336.

38. On peut suivre ainsi, matériellement, à l'aide des planches figurant dans l'ouvrage de l'abbé Frère, les signes phrénologiques de ce développement anthropologique et social des races au cours des sept périodes qui jalonnent sa croissance physique et morale. Dans les premiers stades, la partie postérieure, occipitale du crâne est la plus développée. Cette zone correspond, pour la phrénologie, au siège des instincts. Puis la zone centrale, située sur les os pariétaux et temporaux, s'affirme. Les sentiments résident dans cette aire. Enfin, le front prend son essor dans les derniers stades du développement social: les facultés intellectuelles et réflectives dominent alors l'organisation cérébrale de l'homme.

39. Alphonse ESQUIROS, "Principes de la philosophie de l'Histoire, par l'abbé Frère ", livraison du 9 août 1840, La France littéraire. Littérature-science-arts, nouvelle série, neuvième année, tome II, 1840, p. 184.

40. Alphonse ESQUIROS, Les fastes populaires..., tome deuxième, Paris, 1852, pp. 12-13.

41. Idem, p. 13.

42. Idem, pp. 9-10.

43. Alphonse ESQUIROS, « Des études contemporaines sur l'histoire des races », art. cit., p. 983.

44. Alphonse ESQUIROS, Les Fastes populaires..., tome quatrième, Paris, 1853, p. 2.

45. Alphonse ESQUIROS, «Philosophie du blason. II ", L'Artiste. Revue de Paris. Beaux-arts et belles lettres, I série, Tome IX, Paris, 1847, 1 livraison du 23 mai 1847, p. 179.

46. Victor Hugo explique : «Pour qui sait le déchiffrer, le blason est une algèbre, le blason est une langue. L'histoire entière de la seconde moitié du Moyen Âge est écrite dans le blason, comme l'histoire de la première moitié dans le symbolisme des églises romanes. Ce sont les hiéroglyphes de la féodalité après ceux de la théocratie ». Victor HUGO, Notre-Dame de Paris, 1831, dans Les romans de Victor Hugo, tome III, Lausanne, éditions Rencontre, 1959, p. 162.

47. Alphonse ESQUIROS, «Philosophie du blason. I ", L'Artiste. Revue de Paris. Beaux-arts et belles lettres, I série, Tome IX, Paris, 1847, 1 livraison du 9 mai 1847, p. 148.

48. Alphonse ESQUIROS, «Le musée de Gall », livraison de mai 1843, Revue de Paris, quatrième série, année 1843, tome dix-septième, Paris, 1843, p. 42.

49. Claude-Henri de SAINT-SIMON, «VI Lettre», L'Organisateur. 1 livraison, édition considérablement augmentée, tome premier, Paris, 1820, p. 72.

50. Alphonse ESQUIROS, Introduction, dans Dr. J. Franklin, La vie des animaux. Histoire naturelle, biographique et anecdotique des animaux. Ouvrage entièrement nouveau, traduit de l'anglais par A. Esquiros, tome I, Paris. Hetzel, 1859, p. 8. 
51. Alphonse ESQUIROS, Les Fastes populaires..., tome premier, Paris, 1851, Introduction, pp. XXIIXXIII.

52. Augustin Thierry souligne : « Nous croyons être une nation, et nous sommes deux nations sur la même terre, deux nations ennemies dans leurs souvenirs, inconciliables dans leurs projets : l'une a autrefois conquis l'autre; et ses desseins, ses vœux éternels sont le rajeunissement de cette vieille conquête énervée par le temps, par le courage des vaincus et par la raison humaine ». Augustin THIERRY, Dix ans d'études historiques, 1835, réédition, Paris, Firmin-Didot, 1883, pp. 257-258.

53. Augustin THIERRY, Considérations sur l'histoire de France, 1840, dans Euvres complètes, nouvelle édition revue et corrigée, tome IV, Paris, Furne, 1846, p. 117.

54. Alphonse ESQUIROS, Les fastes populaires..., tome deuxième, Paris, 1852, p. 45.

55. François Guizot soutient : "La révolution a été une guerre, la vraie guerre, telle que le monde la connaît entre peuples étrangers. Depuis plus de treize siècles la France en contenait deux, un peuple vainqueur et un peuple vaincu. Depuis plus de treize siècles le peuple vaincu luttait pour secouer le joug du peuple vainqueur. Notre histoire est l'histoire de cette lutte. De nos jours une bataille décisive a été livrée. Elle s'appelle la révolution ». François GUIZOT, Du gouvernement de la France depuis la Restauration, et du ministère actuel, Paris, Ladvocat, 1820, pp. 1-2.

56. Alphonse ESQUIROS, Les Fastes populaires..., tome premier, Paris, 1851, Introduction, p. II.

57. Il écrit ainsi à Madame de Circourt, en 1850, dans une lettre d'une cruelle lucidité : «J'ai pu me remettre à mon travail, à cette toile de Pénélope, commencée il y a six ans et dont je ne vois pas encore le bout. A toutes les causes de lenteur qui existaient pour moi, notre déplorable révolution en a joint une autre qu'il me faut combattre, c'est le doute sur l'histoire de France telle qu'elle m'apparaissait naguère et que j'aimais à la construire dans un ordre que je croyais être le plan même de la providence. Maintenant je ne la comprends plus, le présent a bouleversé mes idées sur le passé et à plus forte raison sur l'avenir ; j'ai perdu ma foi historique et, chose que je n'aurais jamais cru, ma foi politique s'en va ». Augustin THIERRY, Lettre à Madame de Circourt, le 13 janvier 1850, Bibliothèque de l'Arsenal, [Ms. 13522/3].

58. Alphonse ESQUIROS, «Philosophie politique. Des origines de la nation française ", L'Artiste. Revue de Paris. Beaux-arts et belles lettres, I série, Tome V, novembre 1845 à février 1846, douzième livraison du 18 janvier 1846, p. 184.

59. Alphonse ESQUIROS. Les Fastes populaires..., tome premier, Paris, 1851, p. 14.

60. Idem, pp. 32-33.

61. Idem, Introduction, p. XXIII.

62. Idem, Introduction, pp. XXIII-XXIV.

63. Idem, Introduction, p. xxv.

64. Alphonse ESQUIROS, Les Fastes populaires..., tome deuxième, Paris, 1852, p. 65.

65. Idem, pp. 53-54.

66. Idem, p. 23.

67. Idem, p. 24.

68. Alphonse ESQUIROS, « Des études contemporaines sur l'histoire des race », art. cit., p. 1002.

69. Cette idée capitale est en effet développée par Victor Courtet de l'Isle, bien avant Esquiros, dans son livre, La Science politique fondée sur la science de l'homme, ou Étude des races humaines sous le rapport philosophique, historique et social, Paris, Arthus Bertrand, 1837, XV-400 p.

70. Alphonse ESQUIROS, «Du mouvement des races humaines. Cours de M. Serres », Revue des deux mondes, tome dixième, quinzième année, nouvelle série, 1845 , tome II, livraison du $1^{\mathrm{er}}$ avril 1845, p. 165.

71. Alphonse ESQUIROS, Les fastes populaires..., tome deuxième, Paris, 1852, p. 55.

72. Voir le texte d'Étienne SERRES, «Rapport sur les résultats scientifiques du voyage de circumnavigation de l'Astrolabe et de la Zélée ", Compte rendu des séances de l'Académie des sciences, Tome XIII, $2^{\mathrm{e}}$ semestre, 1841, no 13 , séance du lundi 27 septembre 1841, pp. 643-651. 
73. Alphonse ESQUIROS, Les fastes populaires..., tome deuxième, Paris, 1852, p. 61.

74. Idem, p. 61.

75. Idem, p. 60.

76. Idem, pp. 60-61.

77. Alphonse ESQUIROS, Charlotte Corday, ouv. cit., p. 79.

78. Idem, pp. 63-64.

79. Idem, p. 64

80. Alphonse ESQUIROS, Les vierges martyres, 1842, troisième édition, Paris, P. Delavigne, 1846, p. 33.

81. Alphonse ESQUIROS, «Philosophie politique. Du droit de jambage », L'Artiste. Revue de Paris. Beaux-arts et belles lettres. IV ${ }^{\mathrm{e}}$ série, Tome $\mathrm{V}$, novembre 1845 à février 1846, quinzième livraison du 8 février 1846, p. 234.

82. Alphonse ESQUIROS, Les fastes populaires..., tome deuxième, Paris, 1852, p. 63.

83. Alphonse ESQUIROS, « Philosophie politique. Du droit de jambage », art. cit., pp. 234-235.

84. Alphonse ESQUIROS, "Du mouvement des races humaines. Cours de M. Serres", art. cit., p. 168.

85. Le texte de Charles GAILLARD, Fusion ou L'histoire des peuples expliquée d'après les lois de la physique et de la chimie ; suivi de la France et Louis-Napoléon Bonaparte, Paris, Garnier frères, 1848, 24 p., offre une belle illustration de cette idée défendue par Esquiros.

86. Michel FOUCAULT, « Naissance de la biopolitique. Introduction au cours de l'année 1979 du Collège de France, enregistrement public du 10 janvier 1979 ». De la gouvernementalité. Introduction aux cours des années 1978 et 1979, Paris, éditions du Seuil, 1989.

87. Edouard BONNET-DUVERDIER, "Science et socialisme", L'Homme. Journal de la démocratie universelle, Jersey, $\mathrm{n}^{\circ}$ 8, mercredi 18 janvier 1854 (non paginé).

\section{RÉSUMÉS}

Alphonse Esquiros systématise, dans les Fastes populaires, 1851-1853, ses réflexions antérieures pour fonder une nouvelle philosophie de l'Histoire susceptible de rendre compte de la révolution de 1848. Démontrant la répétition de la Nature dans l'Histoire, il jette les bases d'une philosophie naturaliste du progrès humain. En effet, la dynamique du perfectionnement animant l'Univers, se déploie, en abrégé, dans les races humaines, monades déterminant, comme le démontrent les travaux de l'abbé Frère, le progrès nécessaire des sociétés. Il applique ce schéma à l'histoire nationale en recherchant dans les races l'origine et les secrets de l'Histoire de France. Il montre, en révisant le canevas historique proposé par les historiens bourgeois, le mouvement lent de libération des races asservies. La Révolution française émancipa la classe bourgeoise, de race romaine, mais perpétua à son profit le système de domination exercé sur la classe ouvrière, de sang gaulois. La Seconde République marque avec éclat sa volonté de libération, entamée depuis des siècles, par la dialectique des croisements. À partir de ces considérations historiographiques, la pensée d'Esquiros invite enfin à explorer l'ambition scientifique des doctrines socialistes de cette époque.

Anthropology and progress in the philosophy of history of Alphonse Esquiros. The system of Les fastes populaires. Alphonse Esquiros systematizes in his Fastes populaires, 1851-1853, his previous thoughts oriented toward the establishment of a new philosophy of history able to 
account for the revolution of 1848. In showing the rehearsal of the laws of nature in history, he establishes the bases for a naturalistic philosophy of human progress. Indeed, the dynamics of perfection fulfilled in the Universe, are deployed, in brief, in the human races, which are monads defining, as the works of abbé Frère show, the necessary progress of human societies. He applies this scheme to national history in researching in the human races, the origins and secrets of French history. He shows, in revising the historical canvas proposed by bourgeois historians, the slow movement of liberation undertaken by subject races. The French Revolution emancipated the bourgeois society, of Roman origin, but perpetuated to its own profit the system of domination exercised on the working class, of Gallic blood. The Second Republic illustrates with brillance its desire for freedom, initiated over the centuries by the dialectic of racial mixtures. Starting from these historiographical considerations, the thought of Esquiros invites us to explore the scientific ambition of the socialist doctrines of the period.

INDEX

Mots-clés : Socialisme, Histoire intellectuelle, Races, Historiographie

\section{AUTEUR}

\section{LOÏC RIGNOL}

Doctorant à l'Université Paris VIII, sa recherche porte sur l'anthropologie et le socialisme en France dans la première moitié du XIX ${ }^{\mathrm{e}}$ siècle.

l.rignol[at]libertysurf.fr 